\title{
Interferometric heat-load sensing of a high power solid laser medium
}

\author{
M. Baumgart, C. Glassl, A. Tortschanoff, G. Kroupa \\ CTR Carinthian Tech Research AG \\ Europastr. 4, A-9524 Villach, Austria \\ marcus.baumgart@ctr.at
}

\section{Introduction}

Compact and reliable high-power lasers for applications in rough environments are increasingly demanded by industry. Originally designed for laser ignition [1], CTR's HiPoLas ${ }^{\circledR}$ diode-pumped solid state (DPSS) laser (Fig. 1) combines the advantages of high energies per pulse (up to $40 \mathrm{~mJ}$ ) and long lasting operation in a compact, temperature and vibration resistant housing. Achieving high energy densities in such a miniaturized layout requires in depth understanding of the processes occurring during pumping in the laser cavity. A novel, Fabry-Perot-interferometer based, sensing scheme now enables such in situ measurements in high-energy laser rods.

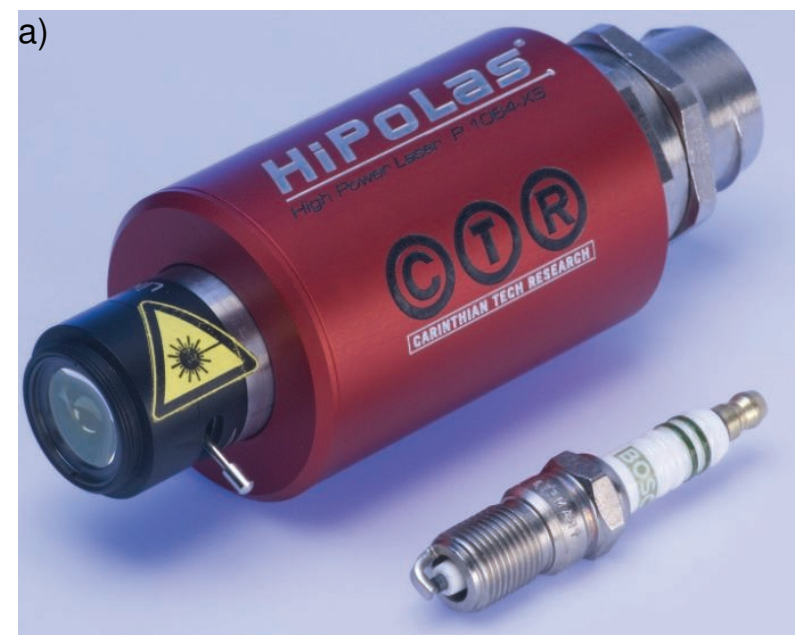

b)

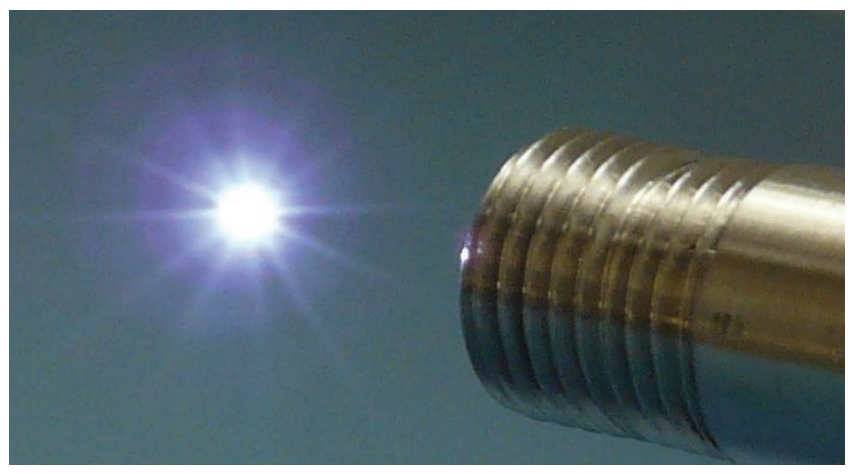

Fig. 1: (a) CTR HiPoLas $\AA^{\circledR}$ laser head used for ignition in engines and conventional electrical spark plug; (b) lonization-driven plasma in air generated by focused high power laser beam (figures reproduced from CTR HiPoLas ${ }^{\circledR}$ datasheet).

\section{Experimental setup and results}

The core component of the specially adapted interferometric measurement setup is a side-pumped monolithic Nd:YAG/Cr:YAG laser rod (Fig. 2b). Special end-surface coatings let the laser rod solely form the laser cavity [2]. High power laser diodes situated in the pump chamber around the laser rod deliver the necessary pump energy for laser beam creation. To measure the pumping effects on the laser rod with appropriate time resolution, an additional intensity-stabilized $632.8 \mathrm{~nm} \mathrm{HeNe}$ analysis laser beam is coaxially coupled through the laser crystal (Fig. 2a).

Multiple internal reflections of the analysis beam occur at the laser crystal's highly parallel end faces. The interference pattern of the transmitted radiation changes with the cavity temperature [3]. The pattern is recorded with a CCD beam camera. Laser rod length changes causes by temperature variation thus can be detected with high resolution.

In the used setup, temperature and thus interference changes (Fig. 3b \& Fig. 3d) could be initiated by both well controlled coolant temperature changes (Fig. 3a) and short (250 $\mu \mathrm{s}$ ) high energy pump pulses (Fig. 3c). To follow the highly transient interaction dynamics during one pump pulse the temperature was varied simultaneous by both methods. The result is a sinusoidal intensity pattern overlaid with the transient effects of the single pulses (Fig. 3d).

Calculations of several important characteristics based on the interference measurements, including laser rod expansion, energy rise inside the laser cavity and absorption-related heat creation are done in the following section. 
a)

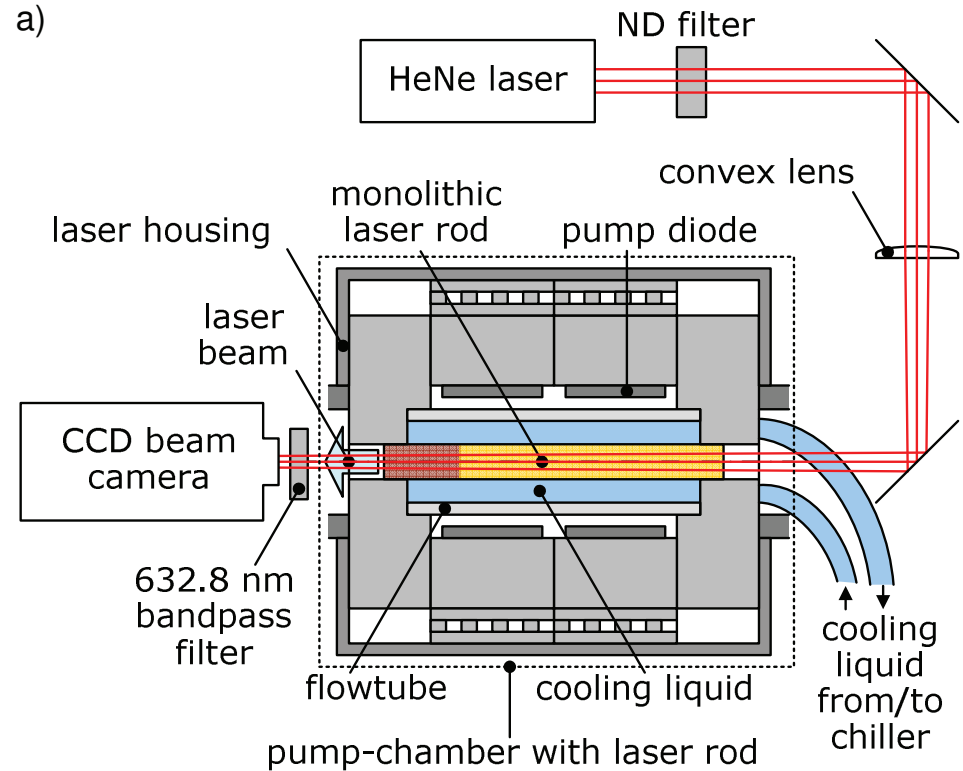

b)

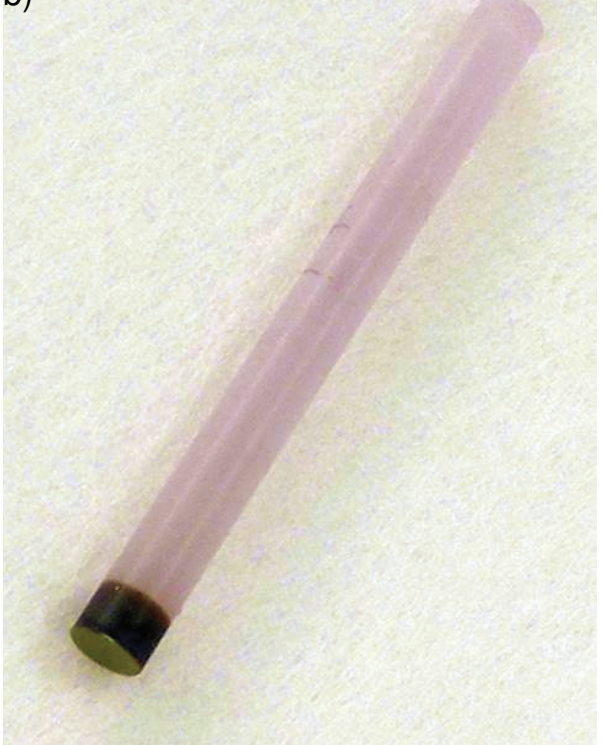

Fig. 2: (a) Experimental setup with CTR pump chamber design for interferometric in-situ thermal expansion and heat input sensing in an endface-coated laser rod; (b) Coated Nd:YAG/Cr:YAG laser rod.

The laser rod length change $\left(\Delta \mathrm{l}_{\text {transient }}\right)$ between an intensity maximum and the following minimum (Fig. 3b) depends only on the wavelength of the analysis laser beam and the refraction index of the Nd:YAG crystal.

$$
\Delta \mathrm{l}_{\text {transient }}=\lambda_{\mathrm{m}} / 4=\left(\lambda / \mathrm{n}_{\mathrm{Nd:YAG}}\right) / 4=(632.8 \mathrm{~nm} / 1.83) / 4=86.44 \mathrm{~nm}
$$

$\lambda_{\mathrm{m}} \ldots$ wavelength in $\mathrm{Nd}: \mathrm{YAG}$ medium, $\lambda \ldots$ wavelength in vacuum,

$\mathrm{n}_{\mathrm{Nd}: \mathrm{YAG}} \ldots$ refraction index of $\mathrm{Nd}: \mathrm{YAG}$

Fig. 4 shows the relevant intensity parameters to calculate the laser rod length change caused by a $250 \mu \mathrm{s}$ long pump pulse. The height $\Delta \mathrm{I}_{\text {transient }}$ between minimum $\mathrm{I}_{\text {transient_min }}$ and maximum $\mathrm{I}_{\text {transient_max }}$ of the slowly changing intensity profile corresponds directly to the above calculated length change $\Delta 1_{\text {transient. }}$.

cooling liquid with $\uparrow$ temperature controlat

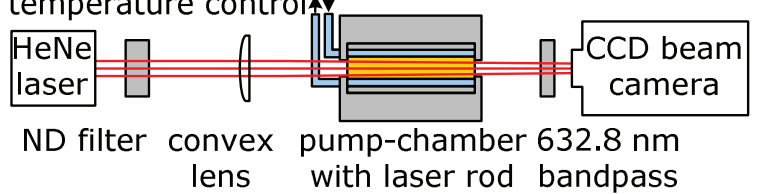

a)

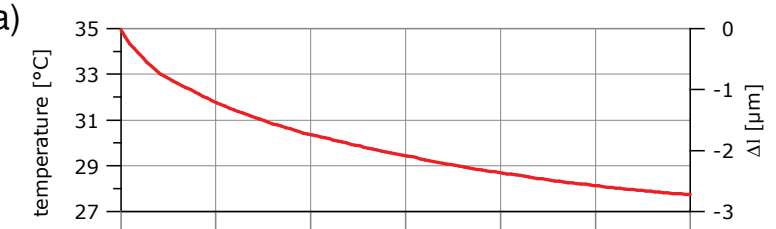

b)

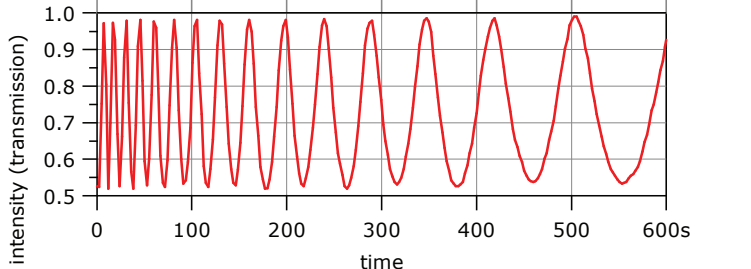

cooling liquid with $\quad$ pulsing of the temperature controlat pump diodes

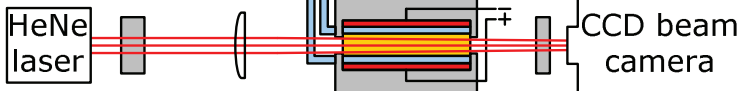

ND filter convex pump-chamber $632.8 \mathrm{~nm}$ lens with laser rod bandpass

c)

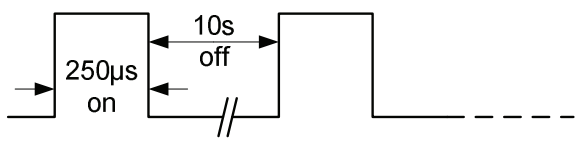

d)

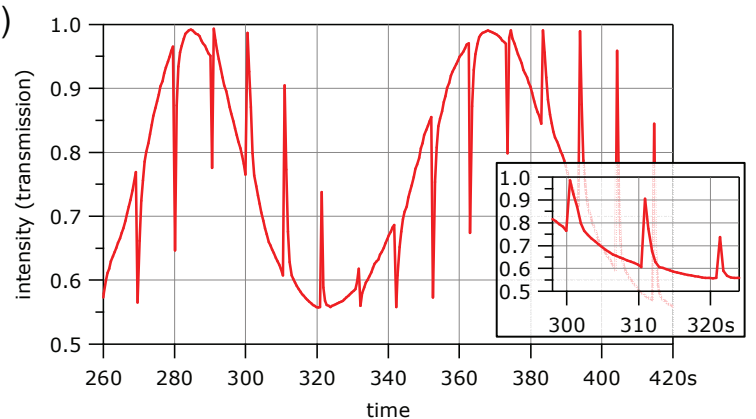

Fig. 3: Effect of changing temperature on the sensor recordings. (a) Temperature vs. time characteristics of coolant liquid and resulting length change of laser rod. (b) Corresponding intensity vs. time profile measured with the CCD beam camera. (c) Transient measurement signals caused by $250 \mu \mathrm{s}$ pump pulses every $10 \mathrm{~s}$ superimposed on a slow sinusoidal intensity variation caused by controlled variation of the coolant temperature. 


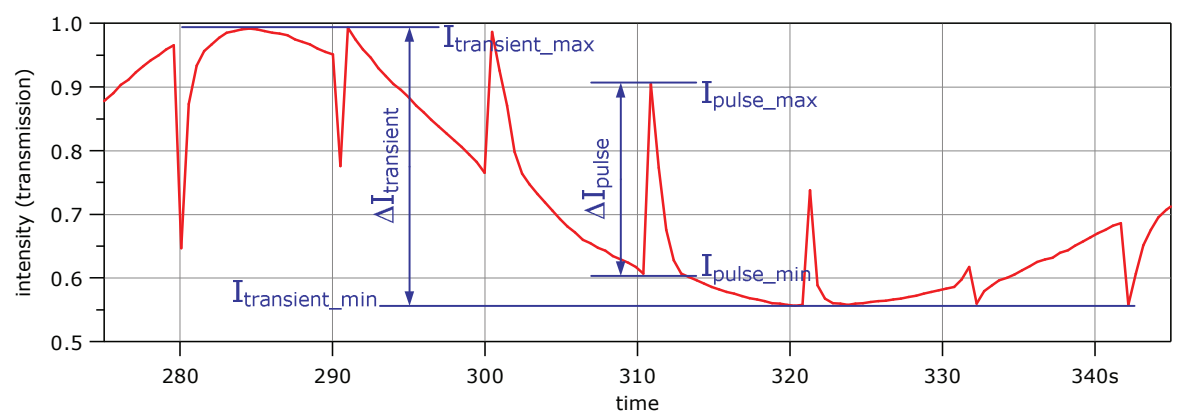

Fig. 4: Determining relevant intensity parameters dor calculation of energy rise inside the laser cavity and absorptionrelated heat creation.

One pump pulse generates an intensity change $\Delta \mathrm{I}_{\text {pulse, }}$, which is smaller than $\Delta \mathrm{I}_{\text {transient }}$. Thus, the length change for one pulse $\Delta \mathrm{l}_{\text {pulse }}$ corresponds directly to $\Delta \mathrm{I}_{\text {pulse }}$.

$$
\Delta \mathrm{l}_{\text {pulse }}=\left(\Delta \mathrm{l}_{\text {transient }} / \Delta \mathrm{I}_{\text {transient }}\right) \cdot \Delta \mathrm{I}_{\text {pulse }}=61.8 \mathrm{~nm}
$$

Considering the thermal expansion coefficient $\left(\alpha=7.8 \cdot 10^{-6} \mathrm{~K}^{-1}\right)$ and the the active pumped length 1 of the laser rod, the following temperature change occurs in the laer rod per one pump pulse.

$$
\Delta \mathrm{T}=\alpha \cdot \Delta \mathrm{l} / \mathrm{l}=0.79 \mathrm{~K}
$$

Finally, the absorbed thermal energy per pump pulse $\Delta \mathrm{Q}$ can be calculated.

$\Delta \mathrm{Q}=\mathrm{c}_{\mathrm{p}} \cdot \mathrm{m}_{\mathrm{p}} \cdot \Delta \mathrm{T}=\mathrm{c}_{\mathrm{p}} \cdot \rho \cdot \mathrm{V} \cdot \Delta \mathrm{T}=171 \mathrm{~mJ}$

$c_{p} \ldots$ specific heat capacity of Nd:YAG $\left(0.59 \cdot 10^{-3} \mathrm{~J} /(\mathrm{kg} \cdot \mathrm{K})\right), \rho \ldots$ density of $\mathrm{Nd}: Y A G\left(4550 \mathrm{~kg} / \mathrm{m}^{3}\right)$

$\mathrm{V}$... cylindrical volume of active pumped area $\left(71 \mathrm{~mm}^{3}\right)$

In our examined setup the laser rod expands $62 \mathrm{~nm}$ during a $250 \mu \mathrm{s}$ pulse, corresponding to a $0.79 \mathrm{~K}$ temperature rise and an absorption-related heat creation of $171 \mathrm{~mJ}$ in the laser rod.

\section{Simulations}

Optical simulations of the pump light absorption corresponding to the experimental setup were done with the ray-tracing software ZEMAX. The important parts of the pump chamber including laser rod, pump diodes, flowtube, cooling liquid and chamber walls were modelled in 3D with apropriate properties. The monte-carlo algorithm based ray tracing software can model scattering effects at each (optical) surface. To speed up calculations scattering was only enabled for the laser rod's side surface. The absorption calculations take also into account the measured pump diode spectrum and the Nd:YAG absorption characteristics. In preperation for the simulations both spectra were recorded in high resolution around our pumping wavelength $(808 \mathrm{~nm})$ and imported into the ray-tracing software (Fig. 5a). The convolution of both spectra was done during ray-tracing by the simulation software.

The biggest simulation uncertainties in the simulation setup arise from non-adequate macroscopic surface scattering models in the software (Fig. 5b) and production related variations of the $\mathrm{Nd}$ dopant concentration. Thus, both of these parameters were varied in combination in the done simulations.

Fig. $5 \mathrm{c}$ presents the simulation results including variation of scattering power and $\mathrm{Nd}$ dopant concentration in the YAG crystal. The 2D plots show the summed absorption from the front to the back side of the laser rod. A qualitatively similar absorption profil with the highest absorption in the middle of the laser rod arises from the simulations in the first, second and fourth plot (low \& medium scattering, two different $\mathrm{Nd}$ concentrations), whereas with the high scattering model and $1.1 \% \mathrm{Nd}$ concentration in the third plot the pump light absorption is very homogenious over the whole laser rod. The resulting absorption values lie in the range between and $181 \mathrm{~mJ}$ and $203 \mathrm{~mJ}$, which corresponds nicely to the above experimental results. The model corresponding best with the real laser rod absorption is hard to identify. Due to the Gaussian beam profile of the laser pulse an absorption profile with the highest absorption in the laser rod's middle and an isotrop absorption decay towards the laser rod side surface border should be preferable for highest energy output, but an experimental verification method is still missing. 
a)

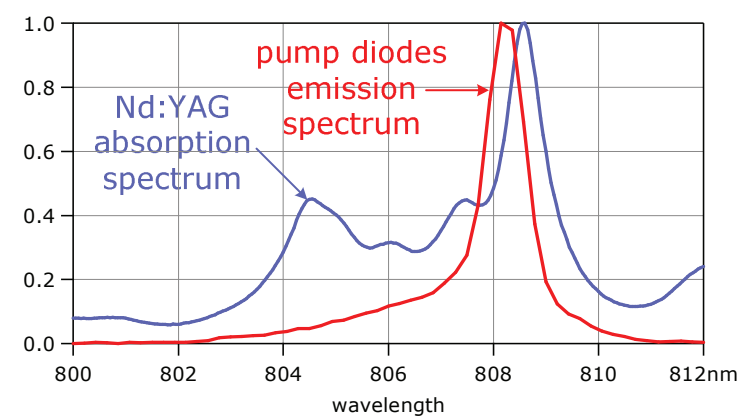

b)

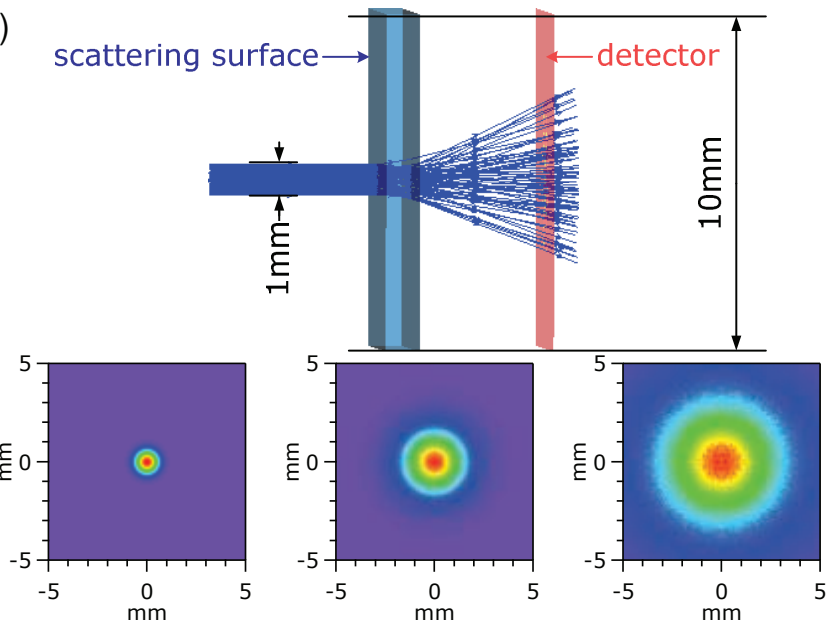

c)

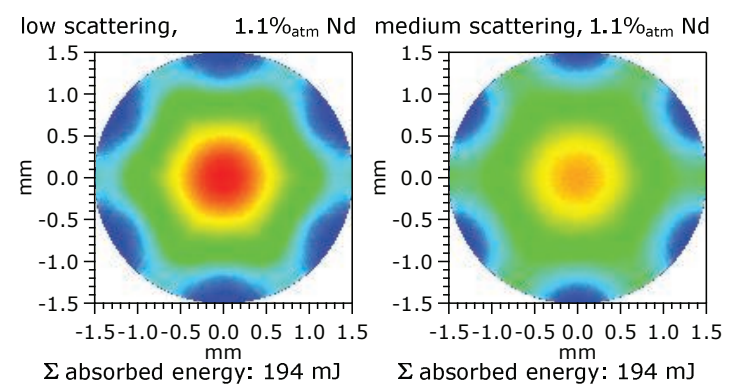

high scattering, $\quad 1.1 \%$ atm $\mathrm{Nd}$ medium scattering, $0.9 \%$ atm $\mathrm{Nd}$
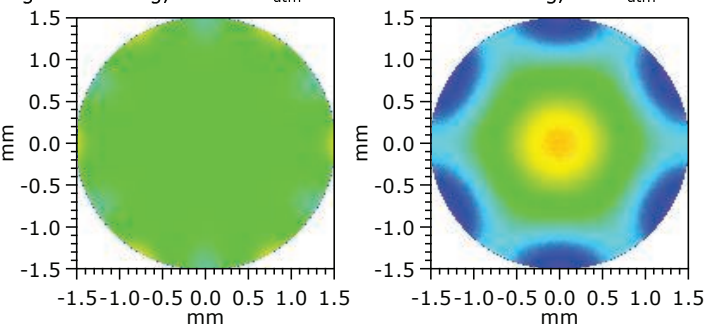

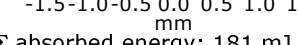

$\Sigma$ absorbed energy: $203 \mathrm{~mJ}$

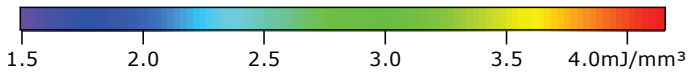

scale: 0 ... 100 [a.u.] scale: 0 ... 14 [a.u.] scale: 0 ... 2.5 [a.u.]

Fig. 5: (a) Measured high resulution spectra of pump diode emission and Nd:YAG absorption (a.u.). (b) Comparison of different scatter fractions defined in the ray-tracing software. (c) Simulated absorption profiles for different laser rod side-surface scattering numbers and Nd dopant concentrations with resulting absorbed energy. The shown absorption profiles were normalized to the measured optical power of the pump diodes.

\section{Conclusion}

With the presented interferometric in-situ monitoring method the dynamics of the lasing element can be resolved down to $\mu$ s pump pulses. Hence, determining the temperature rise in the laser rod, its expansion and the injected heat in real-time is possible. The results are in good agreement with the optical simulations, leading to further optimization of the laser chamber design and cooling layout. Furthermore, the high temporal dynamics give an important insight into the dynamics of the laser pulse transformation and the formation of temporally occurring thermal lens. Thus, the measurements and according simulations will also lead to a better understanding of thermal lens effects and enable the development of improved, energy efficient cooling of the laser rods.

\section{References}

[1] Kroupa G, Franz G, Winklhofer E. Novel miniaturized high-energy Nd YAG laser for spark ignition in internal combustion engines. Opt Eng 2009;48:014202.

[2] Kroupa G, Franz G, Winklhofer E. Solid-State Laser comprising a resonator with a monolithic structure. PCT Appl. WO/2007/143769.

[3] Jacobs SF, Bradford JN, Berthold III JW. Ultraprecise Measurement of Thermal Coefficients of Expansion. Appl Opt 1970;9:2477-2480.

Corresponding author: Marcus Baumgart (marcus.baumgart@ctr.at)

CTR Carinthian Tech Research AG, Europastr. 4, A 9524 Villach, Austria, phone: $+43 / 4242 / 56300212$, fax: $+43 / 4242 / 56300400$ 